\title{
Factors influencing bacterial microbiome composition in a wild non- human primate community in Taï National Park, Côte d'Ivoire
}

\author{
Jan F. Gogarten ${ }^{1,2,3} \cdot$ T. Jonathan Davies ${ }^{1} \cdot$ Jacquelynn Benjamino $^{4} \cdot$ J. Peter Gogarten $\mathbb{1}^{4} \cdot$ Joerg Graf $^{4}$. \\ Alexander Mielke ${ }^{2} \cdot$ Roger Mundry ${ }^{5}$ Michael C. Nelson $\mathbb{1}^{4} \cdot$ Roman M. Wittig $^{2,6} \cdot$ Fabian H. Leendertz ${ }^{3}$. \\ Sébastien Calvignac-Spencer ${ }^{3}$
}

Received: 5 January 2018 / Revised: 4 May 2018 / Accepted: 11 May 2018 / Published online: 28 June 2018

(c) International Society for Microbial Ecology 2018

\begin{abstract}
Microbiomes impact a variety of processes including a host's ability to access nutrients and maintain health. While host species differences in microbiomes have been described across ecosystems, little is known about how microbiomes assemble, particularly in the ecological and social contexts in which they evolved. We examined gut microbiome composition in nine sympatric wild non-human primate (NHP) species. Despite sharing an environment and interspecific interactions, individuals harbored unique and persistent microbiomes influenced by host species, social group, and parentage, but surprisingly not by social relationships among members of a social group. We found a branching order of host-species networks constructed using the composition of their microbiomes as characters, which was incongruent with known NHP phylogenetic relationships, with chimpanzees (Pan troglodytes verus) sister to colobines, upon which they regularly prey. In contrast to phylogenetic clustering found in all monkey microbiomes, chimpanzee microbiomes were unique in that they exhibited patterns of phylogenetic overdispersion. This reflects unique ecological processes impacting microbiome composition in chimpanzees and future studies will elucidate the aspects of chimpanzee ecology, life history, and physiology that explain their unique microbiome community structure. Our study of contemporaneous microbiomes of all sympatric diurnal NHP in an ecosystem highlights the diverse dispersal routes shaping these complex communities.
\end{abstract}

These authors contributed equally: Fabian H. Leendertz, Sébastien Calvignac-Spencer.

Electronic supplementary material The online version of this article (https://doi.org/10.1038/s41396-018-0166-1) contains supplementary material, which is available to authorized users.

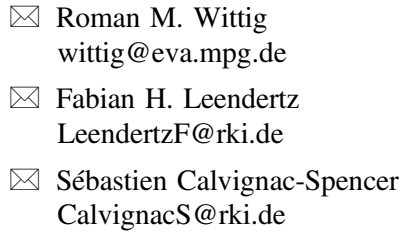

1 Department of Biology, McGill University, 855 Sherbrooke Street West, Montreal, QC H3A2T7, Canada

2 Primatology Department, Max Planck Institute for Evolutionary Anthropology, Deutscher Platz 6, 04103 Leipzig, Germany

\section{Introduction}

Mammalian gut microbiomes represent complex communities. These influence a broad array of processes including a host's ability to access nutrients [1], development and tissue maturation [2], health via pathogen exclusion and immune system priming [3], and even behavior and scent [4]. The importance of this and other microbiome communities (e.g., skin, vaginal, oral) has led to the suggestion

3 Project Group Epidemiology of Highly Pathogenic Microorganisms, Robert Koch Institute, Seestraße 10, 13353 Berlin, Germany

4 Department of Molecular and Cell Biology, University of Connecticut, 91 North Eagleville Road, Unit 3125, Storrs, CT 06269-3125, USA

5 Max Planck Institute for Evolutionary Anthropology, Deutscher Platz 6, 04103 Leipzig, Germany

6 Taï Chimpanzee Project, Centre Suisse de Recherches Scientifiques, B.P. 1303 Abidjan 01, Côte d'Ivoire 
that the holobiont, i.e., the host plus the entirety of its associated microbiomes, is one of the units of biological organization on which natural selection acts [5-7]. These host-microbiome ecosystems are short-lived (with an absolute upper bound being a host's lifetime). However, bacteria are fast-evolving organisms capable of horizontal gene transfer, which provides a means for rapid evolutionary change [8] allowing for strong eco-evolutionary feedback loops between the microbiome, which provides essential ecosystem services, and the host [9].

Despite a lack of strict vertical inheritance of the microbiome, differences in composition of the gut microbiome of hominine species appear to recapitulate their evolutionary relationships, possibly indicating codivergence of microbiomes with their hosts, but perhaps also reflecting the similar gut environments of related host species [10]. Studies on the scale of individual bacterial taxa suggest a number of bacterial lineages have co-diversified with hominines over the last 6 to 12 million years [11]. Interestingly, it appears that diet influences the acquisition of large ancient microbial lineages, while more recently diverged bacterial lineages (more in line with the scale of the host's evolution) appear to correlate more tightly with host phylogenies [12]. The pattern of phylosymbiosis, or co-speciation between hosts and microbiome, can indicate strong vertical inheritance but also horizontal inheritance through host swaps within related hosts or environmental filtering by closely related hosts that select for similar bacteria. Anatomy, physiology, and life history traits covary with host phylogenies, which likely promotes observed patterns of co-divergence between gut bacteria and hosts and deterministic assembly of the gut microbiome. For example, the production of compounds like milk oligosaccharides, immunoglobulins, antimicrobial peptides, and growth factors may promote the colonization and persistence of particular bacteria in a host [13]. A host's diet also provides nutrients and compounds that promote the growth of particular bacteria, while gut anatomy and physiology of a host may further provide conditions amenable to certain types of bacteria (e.g., foregut fermentation provides an environment that facilitates populations of cellulytic bacteria; [14]). Host genotype, physiology, and life history, which all co-vary with host phylogenies, can thus enable a host to foster particular bacteria, but for mammals, these bacteria must still arrive from outside the host, as individuals are born largely microorganism free or are seeded with bacteria in the placenta before birth [15].

In humans, vaginal birth exposes newborns to bacteria which then influence the composition of their microbiome [16], though debate surrounds the long-term consequences of such vertical inheritance of microbiomes and whether it is ultimately a major contributing factor shaping the adult microbiome. After birth, exposure to particular bacteria fosters the establishment of certain bacteria (e.g., through a shared environment or contact with conspecifics). Social contacts provide opportunities for horizontal transmission of bacteria and studies of baboon microbiomes suggested that close social partners have more similar gut microbiomes above and beyond the effect of diet, kinship, and shared environments [17]. In animals that have evolved complex social systems, such as primates, social structure may promote selective exposure to the microbiomes of conspecifics, possibly resulting in a "pan-microbiome" shared by a group or clique.

Interspecies contact through hunting or during formation of mixed host species associations might provide opportunities for grooming, play, and even sexual contact between host species, which is expected to promote sharing of microorganisms between taxa [18]. Sharing an environment might provide another route for exchange of microorganisms. For example, chimpanzees harbor Escherichia coli genetically more similar to those of humans employed in chimpanzee-directed research and tourism than to those of humans from a local village, which might suggest that NHP sharing an environment may be exposed to a similar source pool of bacteria [19]. Sharing an environment or inter-host species contacts may lead to a homogenization of the gut microbiome for primates living in a particular ecosystem, a pattern which was observed for populations of sympatric chimpanzees and gorillas [20]. This suggests that studying geographically isolated populations exposed to different source pools could lead to patterns that appear to suggest coadaptation between microbes and hosts but might be driven by ecological differences and geographic separation among host species.

Here we seek to understand factors influencing microbiome composition in a diverse wild NHP community sharing a common environment. We examined the gut microbiomes of all nine sympatric diurnal NHP species present in Taï National Park, Côte d'Ivoire, and describe the microbiome community structure among individuals within conspecific groups, among social groups within NHP species, and among NHP species in the context of their phylogenetic relationships. First, we tested whether individuals and social groups form biological islands with distinct gut microbiomes and examined how social behavior could mediate both relationships. Second, we contrasted the role of evolutionary history versus diet in shaping NHP species differences in the microbiome and examined whether NHP species maintained distinct microbiomes in a shared environment where they regularly interact.

The NHP community in Taï consists of nine diurnal species: one great ape species, the chimpanzee (Pan troglodytes verus), and eight monkey species, namely three colobine species (Olive colobus-Procolobus verus, Western red colobus-Procolobus badius, King 
colobus-Colobus polykomos) and five cercopithecine species (Sooty mangabey-Cercocebus atys atys, Diana monkey-Cercopithecus diana, Campbell's mona monkey-Cercopithecus campbelli, Lesser spot-nosed monkey-Cercopithecus petaurista, and Greater spotnosed monkey-Cercopithecus nictitans). Colobines are folivores, meaning they feed primarily on leaves and have a specialized physiology facilitating foregut fermentation of their high fiber food sources by a distinct microbiome [14]. In contrast, chimpanzees and cercopithecines are omnivores who consume a more diverse diet of fruits, seeds, insects, leaves, leaf buds, mushrooms, spiders, and smaller vertebrates with an unspecialized gut physiology similar to that of humans [21]. If the microbiome community is primarily structured through co-divergence or preferential hostswitching, we would expect the microbiomes of colobines and cercopithecines to be most similar, as these host clades are sister to each other (Fig. 1b). In contrast, if diet is the primary driver of microbiome community structure, we would expect more omnivorous chimpanzees and
A)
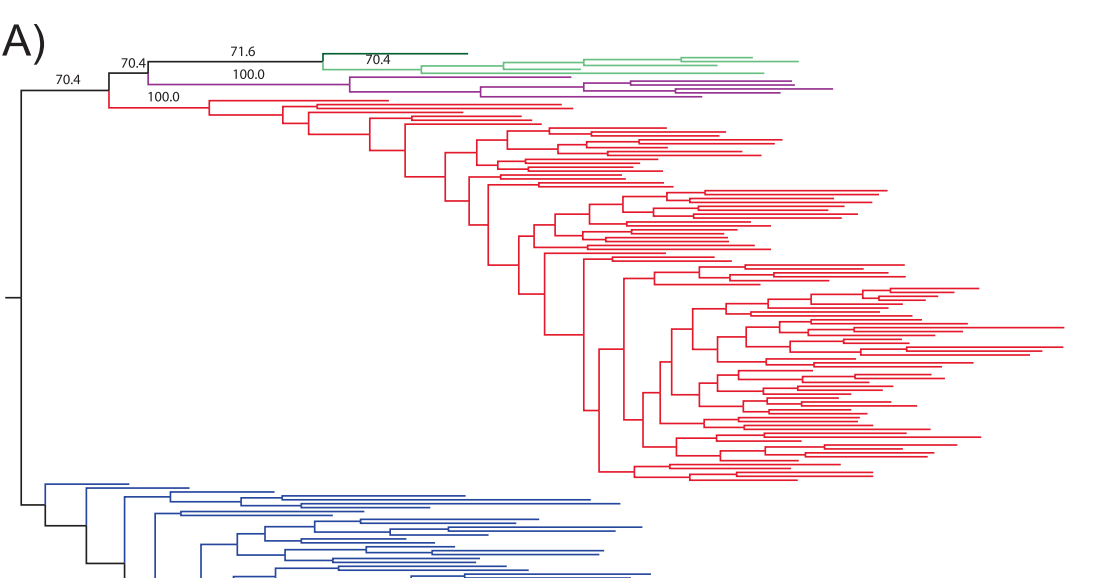

西
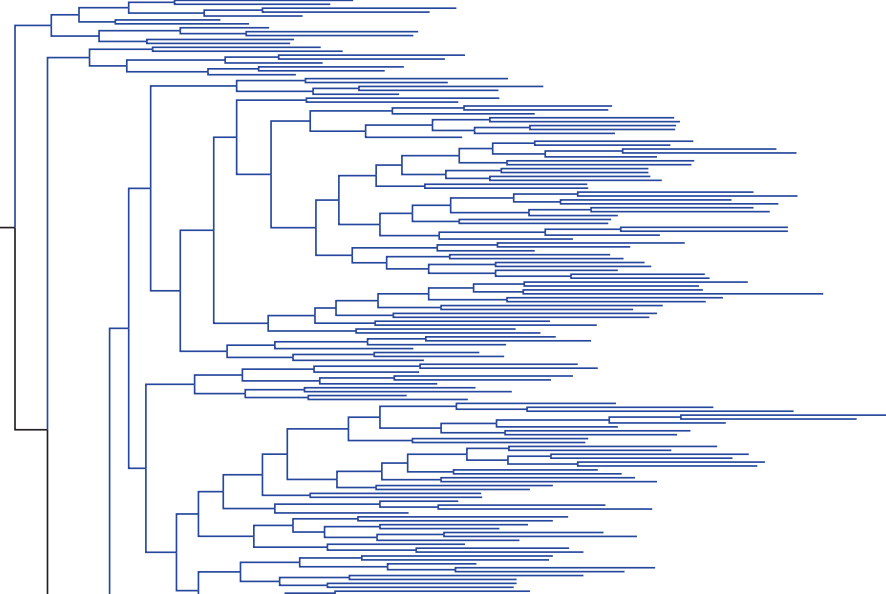

B)

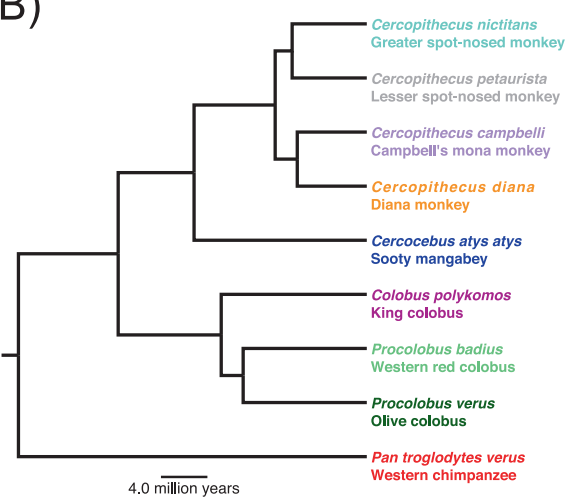

C)

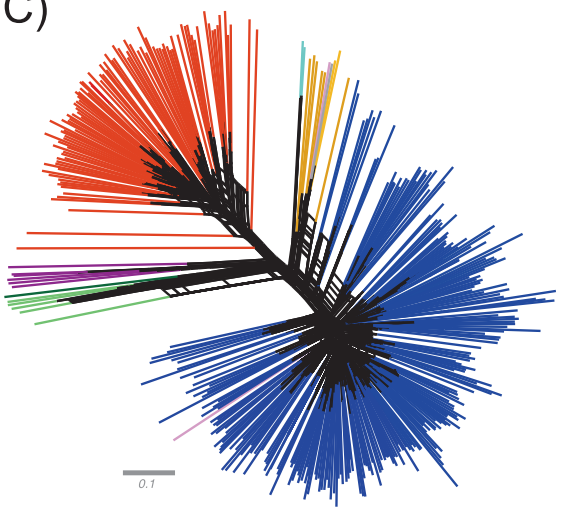

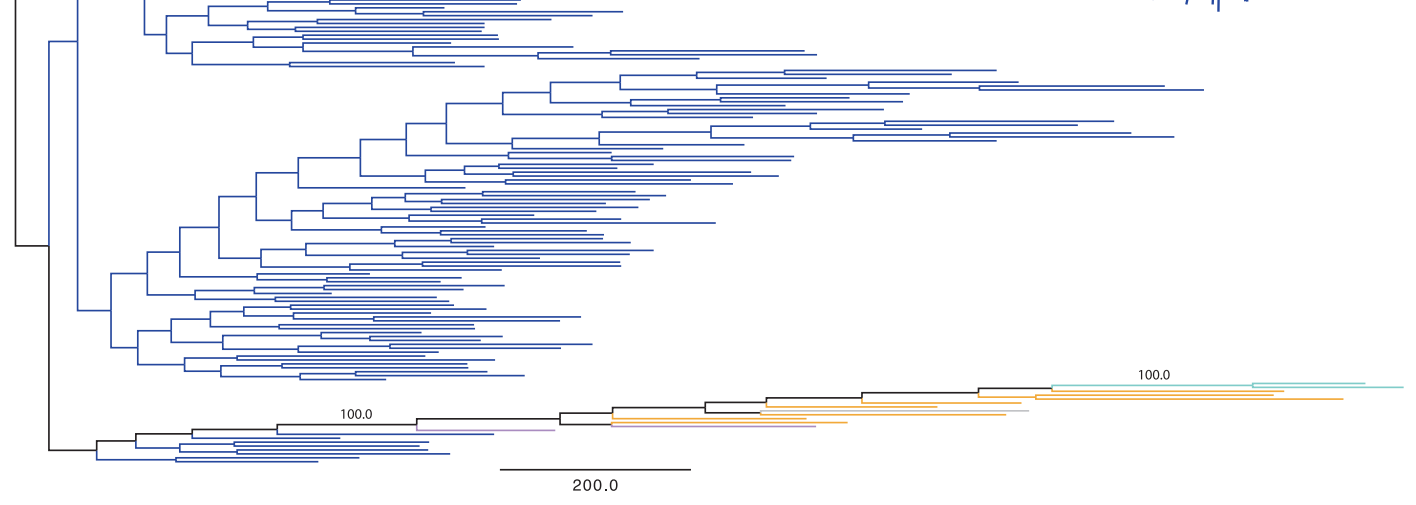

Fig. 1 a Tree of microbiome community structure estimated with heuristic maximum parsimony using the abundance based scores of bacterial abundance as characters. Each terminal branch represents a particular sample, with the colors indicating the NHP species that gave rise to the sample. Black branches indicate internal branches that are shared by different NHP hosts. Bootstrap support is shown for clades and the root position was supported by our TempEst analysis. b Phylogeny of the primate hosts based on 11 mitochondrial and 6 autosomal genes made available through the 10kTrees project [82]. c Unrooted network built using SplitsTree4 and the unifrac dissimilarity matrix as input, with terminal branches colored as in (b) 
cercopithecines that have high overlap in diets, to have more similar microbiomes, with folivorous colobines, who are foregut fermenters, distinct from both chimpanzees and cercopithecines. Our study system also includes a hunter-prey relationship, namely chimpanzees regularly predating colobines, particularly red colobus, including the regular consumption of their intestines [22, 23]. Such trophic interactions have previously been shown to influence the transmission of retroviruses between NHP species [18]. If hunting provides a dispersal route for gut microbes between prey and hunter, we would also expect chimpanzees and colobines to have partially overlapping gut microbiomes, with those of sooty mangabeys and other cercopithecines being more distinct.

\section{Methods}

\section{Site and primary study groups}

Our study was conducted in Taï National Park, Ivory Coast, which consists of $4540 \mathrm{~km}^{2}$ of evergreen rainforest. Two habituated species (i.e., where individual animals are used to human presence and are regularly followed by researchers) of NHP inhabit the study area of the Taï Chimpanzee Project: a group of sooty mangabeys (named the Audrenissrou group) and three neighboring groups of chimpanzees (named the North, South, and East group). The Audrenissrou mangabey group was habituated starting in November 2012, while the chimpanzee groups have been under observation since 1979 [23].

\section{Sample collection}

Fecal sample collection mainly targeted the habituated groups of chimpanzees and mangabeys, but researchers opportunistically collected samples from an additional seven unhabituated monkey species and a neighboring group of mangabeys (Table 1). Fecal samples $(N=380$; Table 1) were stored either by immediately mixing ca. $1 \mathrm{ml}$ feces with an equal volume of RNAlater or ca. $2 \mathrm{ml}$ of feces were kept cool in a thermos in the field and put into liquid nitrogen upon return to the field laboratory. For habituated animals, collection occurred immediately after defecation, while for unhabituated animals, samples were collected once a group was detected and had moved on, collecting a limited number of samples to avoid repeated sampling of individuals. Samples stored in RNA later were homogenized by mixing vigorously and stored for five days at ambient temperature $\left(25-30{ }^{\circ} \mathrm{C}\right)$ and then stored in liquid nitrogen until transport on dry ice to maintain $\mathrm{a}<-80{ }^{\circ} \mathrm{C}$ temperature chain. We detected no impact of preservation method on the gut microbiome composition (Fig. S1, Table S1).

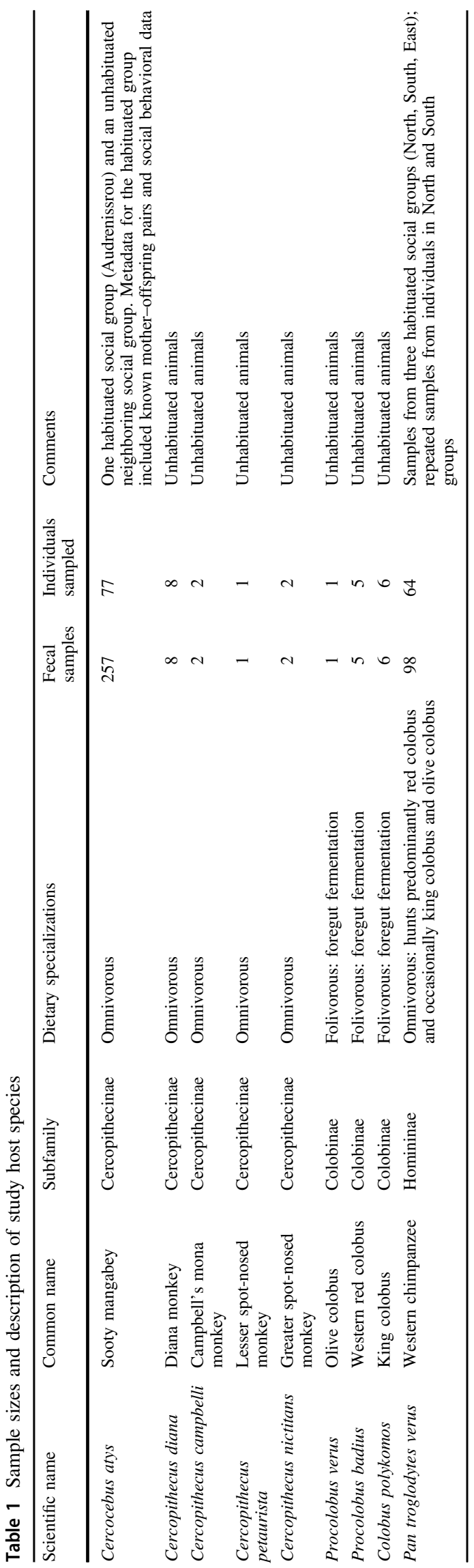




\section{Mangabeys}

To examine turnover in microbiome composition we collected repeated samples from 53 and 26 individuals in the Audrenissrou group in April, May, and June of 2014 and 2015, respectively, with a single additional sample available from September 2013. An additional individual was sampled only once in the 2014 and 2015 sampling periods. To evaluate the importance of the mother-offspring relationship in shaping the gut microbiome, we examined 22 known mother-offspring mangabey pairs. To evaluate the impact of social group membership on the gut microbiome, we collected samples from a neighboring unhabituated group in August and November in 2013, as well as April, May, and June in 2014.

From the Audrenissrou group, we collected social behavioral data using 1-h focal follows of all adults and subadults from 1 January 2014 to 30 June 2015. We continuously collected occurrences of aggressive $(N=1715)$ and grooming $(N=2771)$ behavior, using an ethogram modified from Range and Noë [24]. For focal individuals, we also recorded the nearest neighbor within $1 \mathrm{~m}$ when individuals started feeding or resting $(N=4365)$. We hypothesized that the social behavior preceding and overlapping with sampling would most strongly correlate with microbial composition, so for fecal samples collected between April and June 2014 we analyzed the social behavioral data from January to June 2014, and for the fecal samples collected between April and June 2015 we used behavioral data from January to June 2015. To quantify dyadic grooming, we used a dyadic grooming index: Grooming $(\mathrm{A}+\mathrm{B}) /($ GroomingA + GroomingB-Grooming $(\mathrm{A}+\mathrm{B}))$, where $\operatorname{Grooming}(\mathrm{A}+\mathrm{B})$ is the total time $\mathrm{A}$ and $\mathrm{B}$ spent grooming one another, Grooming $\mathrm{A}$ is the total time spent grooming by individual A and GroomingB is the total time spent grooming by individual B [25]. Dyadic proximity and aggression indices were recorded as for grooming but used the number of observations instead of time [25]. We dropped individuals from an analysis if they had less than 20 observations for a particular social behavior (or in the case of grooming, $20 \mathrm{~min}$ ).

\section{Chimpanzees}

To examine the stability of the microbiome over time we collected repeated samples from individuals in the South ( $N=18$ individuals) and North group ( $N=11$ individuals). We collected a single sample from 28 individuals in the East group to examine whether individuals in neighboring social groups harbored distinct microbiomes. All chimpanzee samples were collected between March and July 2014. Because chimpanzees often prey on colobines, bacterial sequences in chimpanzee feces could represent passaging of the gut microbiome of their prey [26]. To test whether this was a major contributor of $16 \mathrm{~S}$ sequences, we tested for colobine DNA using two colobine specific PCR systems (Colobinae mt 12S rRNA and Colobinae mt CR: described in [27, 28]).

\section{Colobines and other cercopithecine outgroup samples}

To explore cross NHP species variation in gut microbiomes and phylogenetic structure of these microbiomes, we opportunistically collected samples from seven additional sympatric diurnal NHP species. Samples from these unhabituated NHP species were collected in the home range of the Audrenissrou group and were collected during the same period the sooty mangabey samples were collected in April, May, and June of 2014. To confirm the NHP species identity of samples collected from unhabituated primates, we used a PCR targeting the mitochondrial ribosomal $16 \mathrm{~S}$ RNA gene (Forward primer '16Smaml': CGGTTGGGG TGACCTCGGA; Reverse primer '16Smam3': GATGTCC TGATCCAACAT) and the following conditions: $5 \mathrm{~min}$ at $95^{\circ} \mathrm{C}, 42$ cycles $\left[30 \mathrm{~s}\right.$ at $95^{\circ} \mathrm{C}, 30 \mathrm{~s}$ at $64{ }^{\circ} \mathrm{C}, 60 \mathrm{~s}$ at $\left.72{ }^{\circ} \mathrm{C}\right], 10 \mathrm{~min}$ at $72^{\circ} \mathrm{C}[29,30]$. Amplified products were sequenced using Sanger's sequencing and compared to publicly available sequences in GenBank through BLAST [31].

\section{Generating bacterial gut community data}

To characterize the microbiome community, DNA was extracted from samples using the Matrix Stool DNA purification kit (Roboklon). DNA concentrations were quantified using a Syngery HT (Biotek, Winooski, VT) with the Quant-iT PicoGreen kit (Invitrogen, ThermoFisher Scientific). Following Nelson et al. [32] the 16S V4 hypervariable region was amplified using the $515 \mathrm{~F}$ and $806 \mathrm{R}$ primers developed by Caporaso et al. [33]. Amplicons were generated from 5 to $20 \mathrm{ng}$ of extracted DNA in triplicate PCR reactions. These were pooled, quantified, and diluted to 4 $\mathrm{nM}$ prior to pooling amplicons for all samples. Pooled libraries were sequenced on an Illumina MiSeq using a $2 \times$ $250 \mathrm{bp}$ sequencing protocol. Reads were analyzed using an oligotyping method that allowed fine-scale differentiation of bacterial operational taxonomic units, down to a singlenucleotide polymorphism difference between oligotypes [34, 35]. Raw reads were paired, the first 10nt trimmed, with the length of the resulting paired read constrained between $245 \mathrm{nt}$ and 160nt. Zero N-mers and only two errors per read were allowed. Ribosomal sequence variants (RSVs) were inferred after pooling all samples in the dataset using DADA2 [34]. Chimeras were then removed and taxonomy was added to the sequences using the 13-08 release of Greengenes [36]. A maximum likelihood 
phylogeny of the generated oligotypes was generated using an alignment of the $16 \mathrm{~S}$ V4 hypervariable region and a $\mathrm{GTR}+\mathrm{G}+\mathrm{I}$ (Generalized time-reversible with Gamma rate variation) model of sequence evolution with a neighbor-joining starting tree in the phangorn $\mathrm{R}$ package [37]. Reads have been uploaded to European Nucleotide Archive (ENA) study accession number PRJEB18672.

\section{Statistical analyses}

Unless otherwise indicated, statistical analyses were conducted in $\mathrm{R}$ version 3.2.3 [38].

\section{Intraspecific examination of diversity}

We used weighted UniFrac [39] and Bray-Curtis [40] dissimilarity indices to examine pairwise dissimilarity between the bacterial communities of chimpanzee and sooty mangabey microbiomes. Results from the Bray-Curtis dissimilarity index were nearly identical to those of the UniFrac approach and are presented in the Supplementary Material. The UniFrac dissimilarity index incorporates the phylogeny of the bacterial oligotypes by calculating the fraction of shared branch length on the phylogenetic tree between samples [39]. A weighted implementation of the UniFrac index incorporates the abundance of specific oligotypes. Because the weighted UniFrac index can be influenced by sampling effort [39], we first rarefied the data to the minimum sampling effort in a given set of samples being compared. UniFrac dissimilarities range from 0 (i.e., all branches on the bacterial phylogeny are shared between communities) to 1 (no branch length on the bacterial phylogeny is shared between communities) and were calculated using the R package phyloseq [41]. Statistical significance was assessed using Mantel tests and Mantel-like matrix permutation tests [42]. We used data from sooty mangabeys to evaluate the following hypotheses:

1. Microbiomes from an individual sampled at different times are more similar than microbiomes from different individuals.

2. Microbiomes from the same individual are more similar to one another when collected within the same year compared to when collected in different years.

3. Microbiomes from young individuals ( $\leq 3$ years of age at time of sampling) are more similar to that of their mothers than to that of mothers of other offspring.

4. Microbiomes from individuals (of any age) are more similar to that of their mothers than to that of other mothers.

5. Microbiomes from the same social group are more similar to one another than to those from different social groups.
For chimpanzees we used a similar approach to test hypotheses 1 and 5. Mantel tests employed 1000 permutations, including the original data as one permutation. To account for non-independence of samples from the same individual, we permuted subject assignments when comparing between, for instance, groups or years. As a test statistic we used the absolute difference between mean dissimilarities within and between groups, and determined the $P$-value as the proportion of permutations that resulted in a test statistic larger than or equal to that of the original data. To compare similarity of microbiomes of individuals within and between years, we used a Wilcoxon test, because this test incorporates the paired nature of the dataset [43]. We examined the relationship between social behaviors and sooty mangabey microbiomes with Mantel tests using the community dissimilarity matrix and social behavioral matrices. To estimate significance, we determined the proportion of permutations that resulted in an absolute Spearman correlation greater than or equal to that of the original data. Because of observed differences detected between sampling years, we conducted these correlations between community dissimilarity matrices and social behavioral matrices separately for samples collected in 2014 and 2015, and used only samples from adults and subadults as these individuals were targeted by our social behavioral sampling strategy.

\section{Interspecific differences in microorganism abundance}

To investigate whether the presence and abundance of each bacterial oligotype differed between NHP species, we fitted Generalized Linear Mixed Models (GLMM) with a negative binomial error structure and log link function [44, 45], implemented using the function glmer.nb of the R package lme4 [46]. To maximize model stability, we excluded four NHP species for which we had less than five samples and bacterial oligotypes present in $<50$ samples (leaving 1394 oligotypes). To control for seasonal and temporal variation, we focused on samples collected from March to June 2014 $(N=289)$. For each of the remaining oligotypes, we built a model with the number of reads assigned to a particular oligotype as the response variable. Into these models we included NHP species as a fixed effect and individual and social group as random effects. Variation in sampling effort was included as an offset term representing the total number of reads per sample (log-transformed). Models with a dispersion parameter $\geq 1.3$ were excluded from subsequent analyses. To test for the effect of NHP species, we compared the full model with a null model that lacked the fixed effect of NHP species but included the same random effects structure as the full model [47]. We compared null and full models using a likelihood ratio test [48]. We were able to fit a GLMM to 1192 of the bacterial oligotypes. Of these, 24 
had a dispersion parameter $>1.3$ and were not examined further.

\section{Microbiome community structure}

We followed Ochman et al. [10] to compare microbiome community structure between all NHP samples. Working with the rarefied dataset, so that sampling effort was equal, we coded each bacterial oligotype as an ordered multistate character based on orders of magnitude of the number of reads assigned to that taxon (hereafter referred to as the abundance based approach). This character matrix was analyzed using PAUP v4.0b10 and a heuristic maximum parsimony based search with subtree pruning and regrafting (SPR), ACCTRAN optimization with equal weights, and using 250 bootstrap replicates [49]. Tree topology was assessed by examining the mean branch length between clades for each bootstrap replicate. We explored oligotype clustering using parsimony by estimating the best-fitting root with the heuristic residual mean squared function in the program TempEst [50], which minimizes the variance of root-to-tip distances. To further explore clustering of microbiomes, we constructed a network from the pairwise weighted UniFrac distance matrix using SplitsTree4 [51] and examined pairwise UniFrac distances between NHP species.

To examine the phylogenetic structure of NHP fecal microbiome communities we used the mean pairwise phylogenetic distance (MPD) among oligotypes and compared these to a null model based on random assembly from a regional species pool [52]. MPD ranges from 0 to the maximum tree depth, with small values indicating phylogenetic clustering of closely related species and large values indicating phylogenetic overdispersion, i.e., assembly of more distantly related species [52]. To contrast community structure among samples, we estimated standardized effect sizes (SES) by subtracting the mean MPD of 1000 communities assembled randomly from the observed MPD and dividing by the standard deviation of the 1000 randomizations [52]. Positive SES-MPD values indicate overdispersed communities, i.e., communities composed of species that are less related than expected under the null model, while negative values represent under-dispersion, communities composed of species that are more related than expected under the null [52]. We examined variation in SES-MPD across samples using a linear mixed-effects model with a Gaussian error structure and host species as a fixed effect and individual and social group as random effects. We excluded four NHP species for which there were less than five samples. Models were fited based on Maximum Likelihood using the function lmer of the R package lme4 [46], employing the same model diagnostics used for the model testing for bacterial differences between NHP species described above.
Lastly, we examined hierarchical structuring in microbiome communities, i.e., social groups nested within NHP species, mother-offspring pairs nested within groups, and individuals nested within these mother-offspring pairs, by examining the strength of phylogenetic clustering (SESMPD) assuming nulls constructed from nested source pools. If nesting is evident, phylogenetic clustering will get stronger as the source pool becomes more inclusive and more species are added to the bacterial phylogeny. In contrast, if there is no hierarchical nesting, then increasing the source pool will not impact the strength of phylogenetic clustering. We conducted the analyses for both chimpanzees and mangabeys separately. Because chimpanzees uniquely exhibited evidence for phylogenetic overdispersion, we evaluated the mean nearest phylogenetic taxon distance (MNTD) in this NHP species to explore the phylogenetic depth of structuring. In general, SES-MNTD is more sensitive to differences in closely related taxa whereas SESMPD is more sensitive to patterns deeper in the phylogeny [53]. SES-MPD and significance tests were calculated using an implementation in PEZ [54], which draws on the Picante $\mathrm{R}$ package [55].

\section{Results}

We found 3818 oligotypes present in the gut microbiomes of NHP in TNP (Table S2). Of these, 3738 could be assigned to a phylum, with most belonging to Firmicutes (2213) and Bacteroidetes (504) and fewer to Proteobacteria (325), Tenericutes (265), Cyanobacteria (98), Actinobacteria (85), Verrucomicrobia (68), and Spirochaetes (56). A few oligotypes belonged to Euryarchaeota (33), Lentisphaerae (19), Planctomycetes (12), Fusobacteria (11), Acidobacteria (11), Chloroflexi (8), Elusimicrobia (7), Fibrobacteres (4), WPS-2 (3), TM7 (3), Synergistetes (3), Chlamydiae (3), Crenarchaeota (2), and Thermi (2), while only a single oligotype was assigned to each of WS3, Nitrospirae, and Gemmatimonadetes. The core microbiome, here considered as oligotypes found in more than $80 \%$ of individuals of a NHP species, varied between NHP species, though all had significant proportions of Firmicutes and Bacteroidetes (Fig. 2a). Of the 3818 oligotypes, 1481 could be assigned to a previously described genus, with Ruminococcus (180), Prevotella (162), Oscillospira (116), Clostridium (100), Coprococcus (89), Faecalibacterium (51), Blautia (43), Treponema (32), and RFN20 (32) represented by more than 30 known oligotypes. After rarefying the data to the minimum number of reads in a sample (4158), 155 oligotypes were no longer present in any sample, while 1616 oligotypes were specific to a particular NHP species. Only two oligotypes were found in at least one sample in each NHP species (both oligotypes in the 
A)
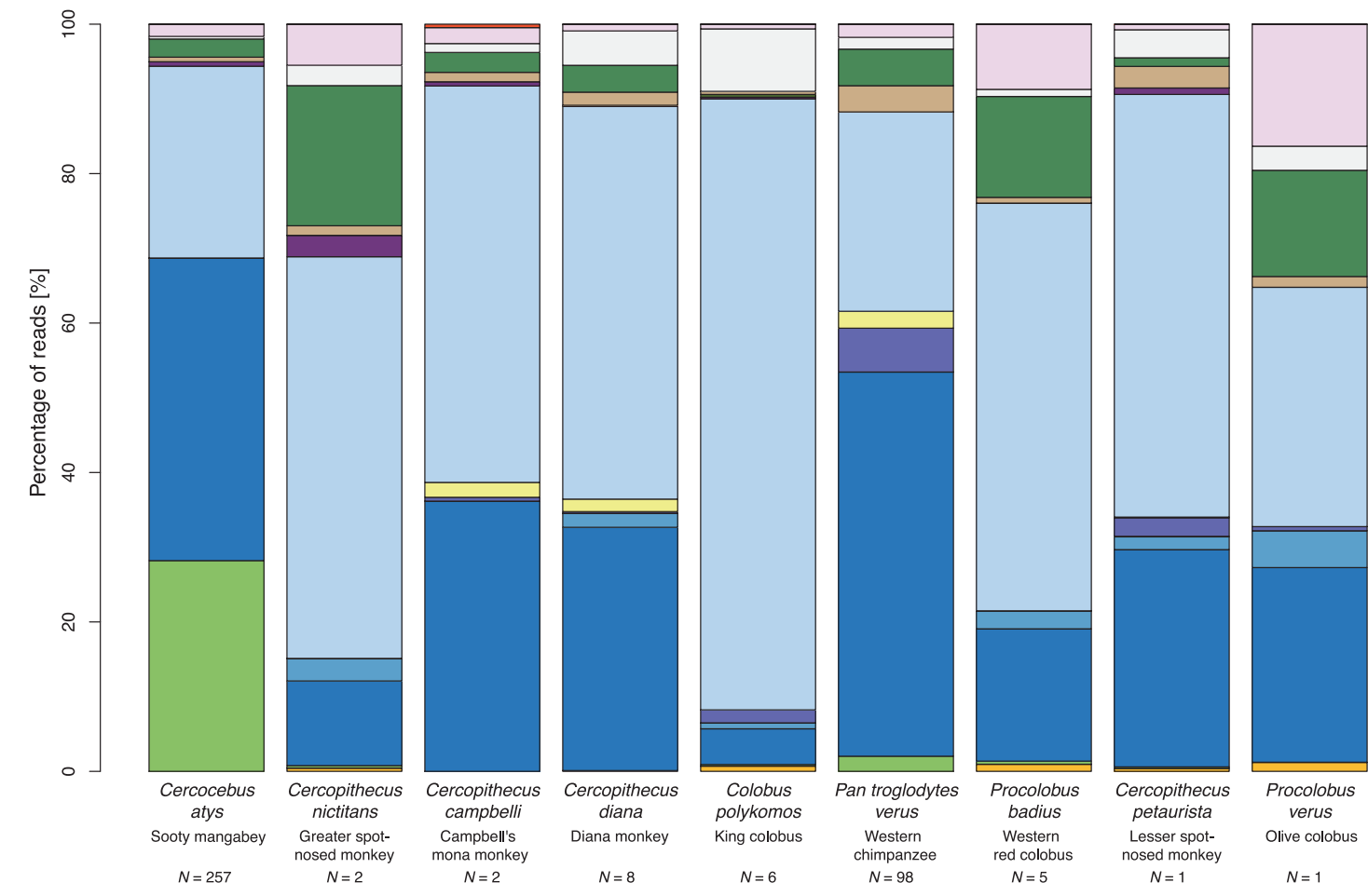

Bacterial phylum $\square$ unknown - Acidobacteria - Actinobacteria - Bacteroidetes $\square$ Cyanobacteria $\square$ Elusimicrobia - Euryarchaeota $\square$ Fibrobacteres $\square$ Firmicutes - Lentisphaerae - Plantomycetes - Proteobacteria Spirochaetes Spirochaetes $\square$ Verrucomicrobia
$\square \square$ $\square$ Verrucomicrob
WPS-2
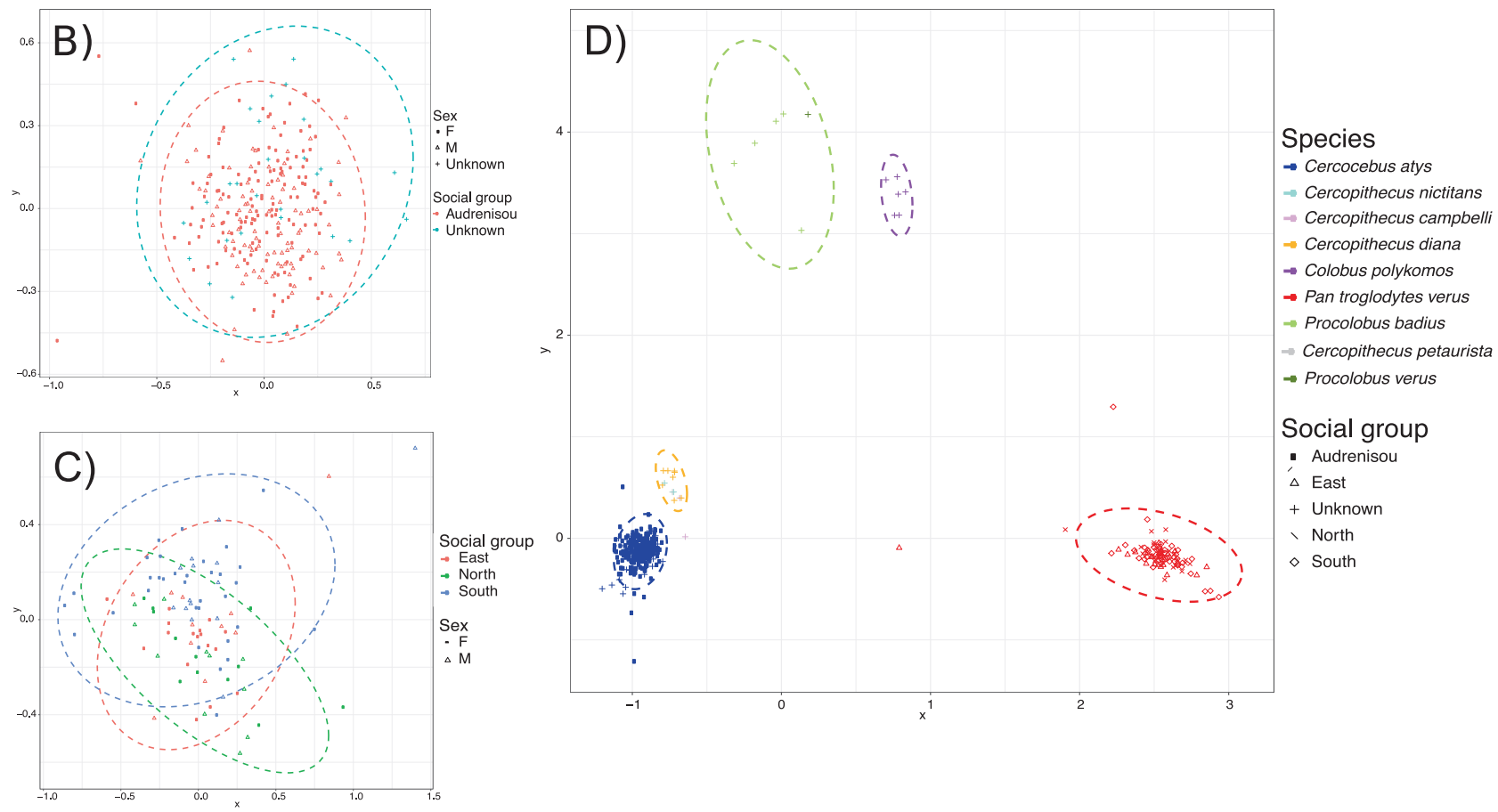

Fig. 2 a Abundance of bacterial phyla for core bacterial oligotypes (i.e., those shared by at least $80 \%$ of individuals in a host species, with the number of reads rarified to the minimum in any sample in the dataset or 4158 reads), shown separately for each NHP species. b Nonmetric multidimensional scaling (NMDS) ordination of mangabey gut bacterial oligotype abundance data by social group (Bray-Curtis distance, stress value $=0.24$; Mantel test: $n_{\text {samples }}=257, n_{\text {individuals }}=87$, $\bar{x}_{\text {different group }}=0.0737, \bar{x}_{\text {same group }}=0.0862, P<0.001$ ). c NMDS ordination of chimpanzee gut bacterial oligotype abundance data
(Bray-Curtis distance, stress value $=0.19$ ) by social group (Mantel test: $n_{\text {samples }}=98, n_{\text {individuals }}=64, \bar{x}_{\text {UniFrac different group }}=0.439, \bar{x}_{\text {Unifrac }}$ same group $=0.408, P<0.001)$. d NMDS ordination of gut bacterial oligotype abundance data by host species (Bray-Curtis distance, stress value $=0.068$ ) of gut bacterial oligotype abundance data by NHP species. Ellipses indicate the $95 \%$ confidence ellipse when more than two samples were available for a particular NHP species or social group 
family Ruminococcaceae, one unknown species of the genus Oscillospira and the other of unknown genus).

\section{Intraspecific beta diversity}

Within mangabeys, we found that samples from the same individual were more similar than samples from different individuals (Fig. 3a). This effect was more pronounced when years were analyzed separately (Mantel test; 2014: $n_{\text {samples }}=154, \quad n_{\text {individuals }}=58, \quad \bar{x}_{\text {different }}$ individuals $=0.0744$, $\bar{x}_{\text {same individual }}=0.0549, P<0.001 ; 2015: n_{\text {samples }}=74, n_{\text {indi- }}$ viduals $=27, \quad \bar{x}_{\text {different }}$ individuals $=0.0650, \quad \bar{x}_{\text {same }} \quad$ individual $=$ $0.0588, P<0.001)$. For the 26 individuals sampled in both years, samples from the same individual were more similar when from the same year than when from different years, suggesting turnover over time (Fig. 3b). However, microbiomes of samples collected between years were more similar when from the same individual than from different individuals, suggesting individual differences in microbiome persisted across years (Fig. 3c). Both familial relationships and group membership impacted the gut microbiome. Samples from the same mangabey group were more similar to one another than samples from different groups (Fig. 2b), and bacterial communities from mother-offspring pairs were more similar to one another than to those from offspring and non-mothers (Fig. 3d), though this effect disappeared when we included offspring older than 3 years of age in this analysis (Mantel test: $n_{\text {samples }}=136, n_{\text {mother-offspring pairs }}=22, \bar{x}_{\text {non-mother-offspring pair }}$ $=0.072, \bar{x}_{\text {mother-offspring pair }}=0.696, P=0.387$ ). There was no correlation between dyadic frequencies of grooming, aggression, proximity, or co-occurrence in a subgroup and
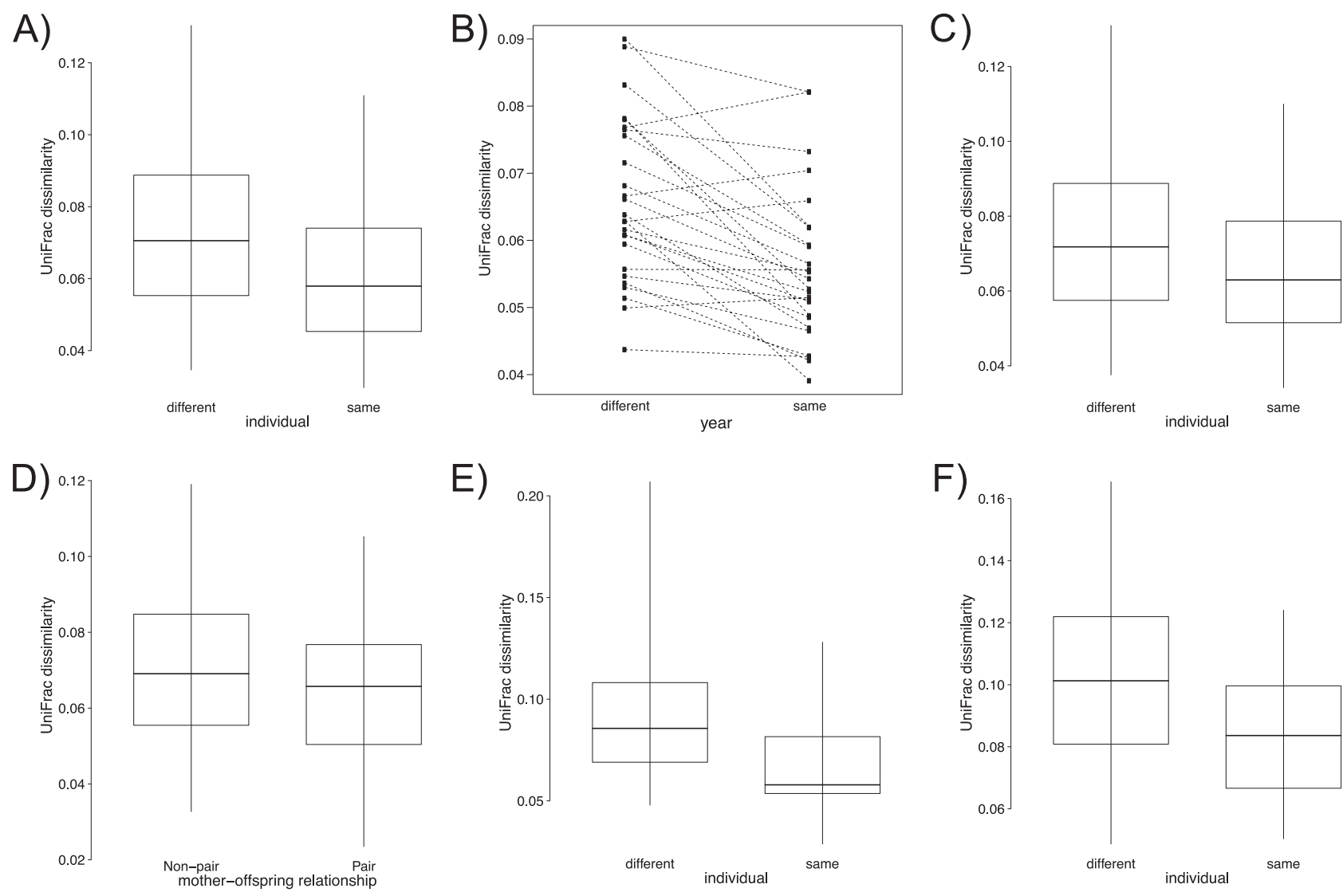

E)

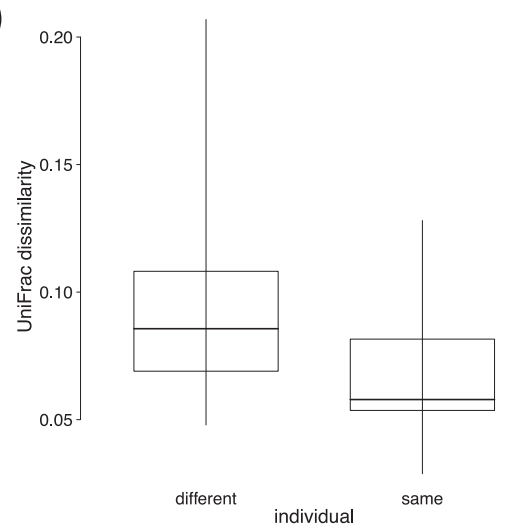

F)

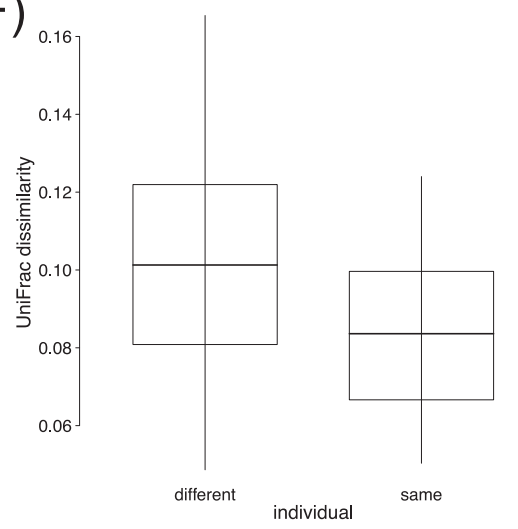

Fig. 3 Comparison of weighted UniFrac dissimilarities between samples from sooty mangabeys in the Audrenissrou group, (a) when stemming from the same or different individuals (Mantel-like permutation test: $n_{\text {samples }}=229, n_{\text {individuals }}=59, \bar{x}$ different individuals $=$ $\left.0.0737, \bar{x}_{\text {same individual }}=0.0610, P<0.001\right)$, (b) between samples of the same individual sooty mangebeys in different or the same years $\left(n_{\text {samples }}=146, \bar{x}_{\text {between sampling year }}=0.0658, \bar{x}_{\text {within sampling years }}=0.0559\right.$; Wilcoxon test, $\left.T^{+}=348, N=26, P<0.001\right),($ c) for the same sooty mangabeys individuals sampled in the same versus different sampling years $\left(n_{\text {samples }}=229, n_{\text {individuals }}=59, \bar{x}_{\text {different individuals different years }}=\right.$ $\left.0.0749, \bar{x}_{\text {same individual different years }}=0.0658, P<0.001\right),(\mathbf{d})$ when stemming from sooty mangebey mother-offspring pairs or from non- mother-offspring pairs (Mantel test: $n_{\text {samples }}=117, n_{\text {mother-offspring pa }}$ irs $=18, \bar{x}_{\text {non-mother-offspring pair }}=0.0705, \bar{x}_{\text {mother-offspring pair }}=0.0637, P$ $=0.033$ ), and (e) between samples from chimpanzees in the South group $\left(n_{\text {samples }}=47, n_{\text {individuals }}=24, \bar{x}_{\text {different individuals }}=0.0933, \bar{x}_{\text {same }}\right.$ individual $=0.0672, P<0.001)$ and (f) chimpanzees in the North group $\left(n_{\text {samples }}=23, n_{\text {individuals }}=12, \bar{x}_{\text {different individuals }}=0.101, \bar{x}_{\text {same individual }}=\right.$ $0.0836, P=0.037$ ) when stemming from the same or different individuals. The middle horizontal line represents the median while the rectangle shows the quartiles and the vertical line represents the 2.5 th and 97.5th percentiles. Dashed lines in (b) indicate the paired nature of the dataset, connecting the dissimilarity for samples from each individual from the same or different sampling years 
Table 2 Spearman rank correlations between social behavior and UniFrac bacterial community dissimilarity in Mangabeys ( $P$-values derived by Mantel tests)

\begin{tabular}{llllll}
\hline & 2014 & & & 2015 \\
\cline { 2 - 3 } \cline { 6 - 6 } Social behavior & $r_{\mathrm{sb}}$ & $P$ & & $r_{\mathrm{sb}}$ & $P$ \\
\hline Grooming & -0.075 & 0.902 & & -0.026 & 0.704 \\
Aggression & -0.002 & 0.515 & -0.015 & 0.549 \\
Proximity & -0.06 & 0.879 & & -0.007 & 0.565 \\
\hline
\end{tabular}

dissimilarity in bacterial community composition (Table 2 and S3).

Chimpanzees showed similar trends: samples from the same individual were more similar than samples from different individuals (Fig. 3e, f), and samples from the three study groups were more similar within than between groups (Fig. 2c). The presence of colobine DNA in a sample did not appear to influence the gut microbiome of chimpanzees, though small sample sizes (6) precluded an in-depth analysis of this factor (Fig. S2).

\section{Interspecific differences in microbiomes}

The abundance of particular bacterial oligotypes was predicted by the host's species (Fig. $1 \mathrm{~d}, n_{\text {samples }}=288$,

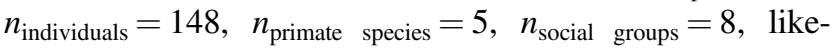
lihood ratio test comparing full and null model, $P<0.05$ for 1150 out of 1168 models). Our maximum parsimony analysis of oligotype abundance provided strong evidence for samples from particular host species forming distinct clades; particularly chimpanzee, colobine, and cercopithecine clades were well supported (Fig. 1a). The Splitstree network built using UniFrac dissimilarity scores showed a similar pattern (Fig. 1c). The chimpanzee, cercopithecine, and colobine clades in the maximum parsimony analysis were all separated by relatively short branches but the shortest distance was between the chimpanzee and colobine clades (Fig. S3). When rooting the tree by minimizing the variance of root-totip distances, the chimpanzee and colobine clades grouped as sisters. To test the robustness of this rooting, we also analyzed the root position in 100 bootstrap pseudo-replicates. Only a single pseudo-replicate supported a chimpanzee outgroup, a relationship that would be predicted by the primate phylogeny. The remaining 99 bootstrap pseudoreplicates were consistent with a chimpanzee-colobine clade, though in four replicates a colobine sample was placed in the cercopithecine clade and for 27 replicates a mangabey sample occurred in the colobine clade. Nearly identical results were observed in a presence-absence based maximum parsimony analysis (see Supplementary Results for details), with the chimpanzee clade clustering more closely with the colobine clade (Fig. S4).
The microbiomes of the majority of samples exhibited strong phylogenetic structure, with phylogenetic clustering most common, with the exception of chimpanzee samples, for which phylogenetic overdispersion was evident (Table 3, S4, S5, Fig. 4; likelihood ratio test comparing full and null model: $\left.\chi^{2}=39.62, \mathrm{df}=4, \quad P<0.001\right)$. Sooty mangabeys additionally showed a signal indicative of nesting, where the strength of clustering increased with the scale of the source pool (Fig. 5a) such that sooty mangabeys had a clustered subset of the bacteria present within all primates, sooty mangabey groups had a clustered subset of the bacteria within the host species, mother-offspring pairs had a clustered subset of those within the group, and individuals a clustered subset of the bacteria within their mother-offspring pair. In contrast, for chimpanzees, increasing the source pool did not expand the phylogenetic breadth of the gut bacterial community; supporting the observation that individuals and groups tended to sample from overdispersed bacterial lineages (Fig. 5b). If anything, phylogenetic overdispersion appeared to increase with an expanded source pool, suggesting these broader scales interspersed oligotypes within clades already represented within the finer sampling rather than introducing phylogenetically distinct bacterial clades. However, by exploring metrics of SES-MNTD, we found some evidence for phylogenetic clustering of tip taxa (Fig. S5); thus, the chimpanzee microbiome appeared to consist of overdispersed clusters of bacterial oligotypes.

\section{Discussion}

We present a study of the gut microbiomes of a diverse community of sympatric, wild, non-human primates (NHP), including a hunter-prey relationship. Results support findings suggesting sympatric wild NHP have individually distinct gut microbiomes despite sharing an ecosystem [56, 57]. We found evidence that both sooty mangabey and chimpanzee social groups maintained a 'pan-microbiome' and that mother-offspring microbiome transmission in sooty mangabeys had an influence on the early life microbiome. During this 'age of assembly', when individuals transition to an adult-like diet and immunological maturity we might expect that social behaviors like grooming and proximity influence the bacterial source pool. In contrast to findings from studies of baboons [17], mangabeys showed no evidence for close social partners having more similar gut microbiomes. Further, we observed incongruence between microbial communities and NHP evolutionary relationships, which contrasts with studies on wild great apes that suggested ape evolutionary relationships were recapitulated by their fecal microbial communities [10]. Contrary to phylogenetic expectations, we found that the 


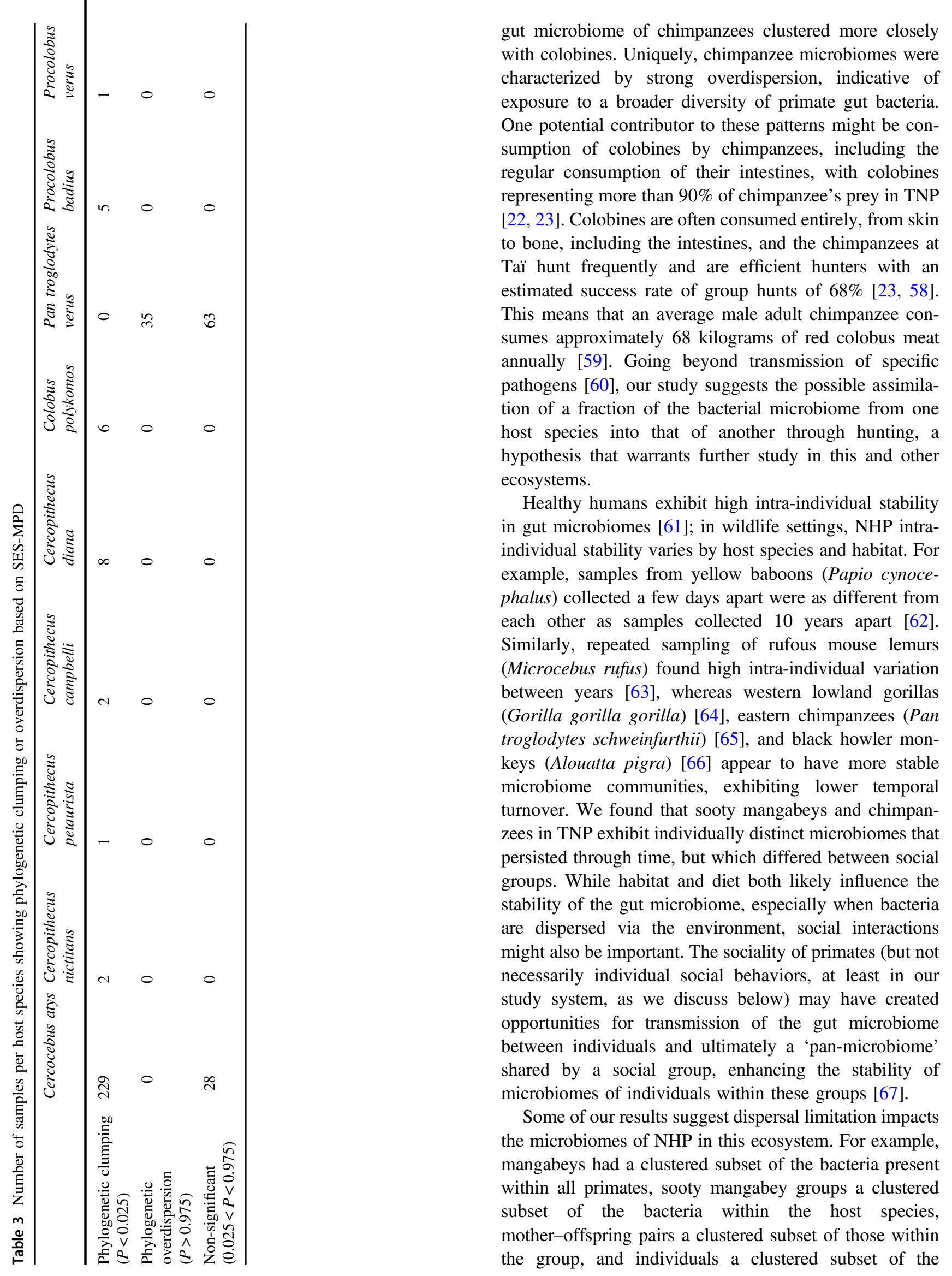




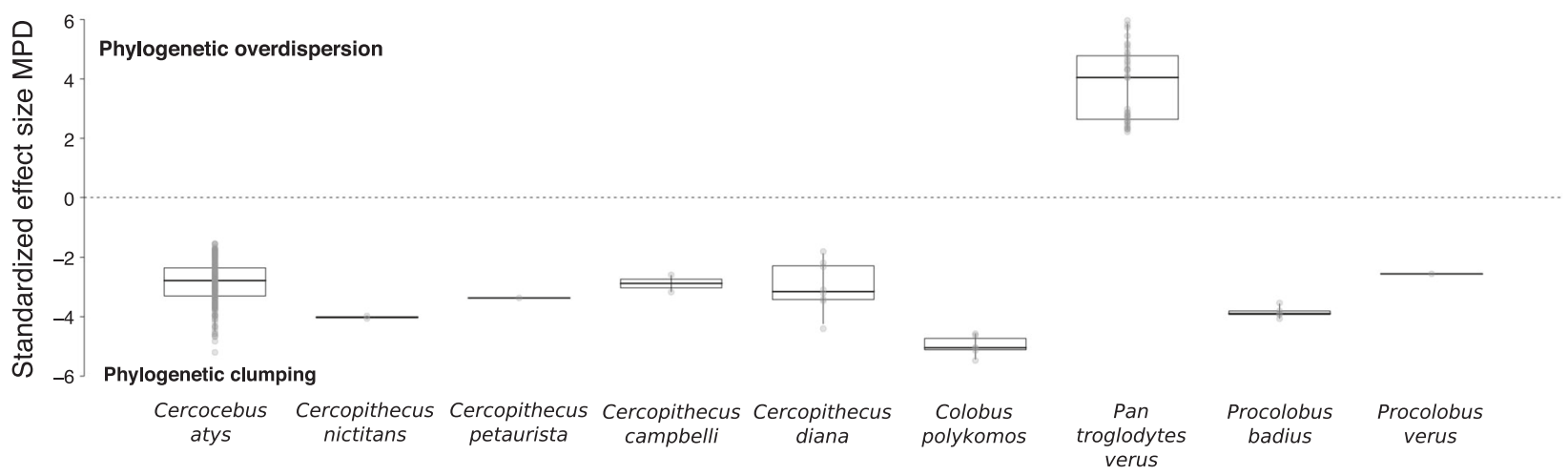

Fig. 4 Standardized effect size of mean phylogenetic distance based on null model simulations of the bacterial community in each fecal sample, separated by host species. The solid middle horizontal line of the rectangles represents the median, the rectangle shows the quartiles, and the vertical line represents the 2.5th and 97.5th percentiles, while the values for each sample are indicated by overlapping gray circles.

bacteria within their mother-offspring pair. In humans, transmission of the microbiome from mothers to infants during vaginal birth shapes an individual's microbiome $[16,68]$, though environmental exposure may shape the later development of the adult microbiome, and these maternal effects may be transitory [69]. Our findings suggest a similar signal in wild NHP, with young sooty mangabeys having more similar microbiomes to their mothers, though this effect disappeared in older individuals as they developed their own distinct microbiome, seemingly from the bacteria present in their social group. In baboons and chimpanzees, the microbiome appears to be transmitted in part by an individual's sociality later in life. More social chimpanzees exhibit more diverse microbiomes [70], and in baboons close social partners exhibited more similar microbiomes [17]. To our surprise, we found no evidence that close mangabeys social partners were more similar in their gut microbiomes, though social group mates did share more similar microbiomes. One potential factor driving this difference to baboons could be that baboon microbiomes exhibit high turnover, even within days [62], and labile microbiomes might be more susceptible to colonization by bacteria. In contrast, sooty mangabey microbiomes were stable over longer periods, and this stability might render social interactions less important for colonization. Nonetheless, there may be a critical period during which dispersal from conspecifics shapes the gut microbiome of sooty mangabeys, but we were not able to detect this with our data.

The different community phylogenetic structures observed in chimpanzees and monkeys in TNP suggest different fundamental processes are shaping the composition of their microbial communities. The majority of NHP microbiomes we examined exhibited significant phylogenetic clustering, whereby closely related bacteria were more
Values above the dashed line are those exhibiting phylogenetic overdispersion (i.e., communities composed of bacterial oligotypes that are less related than expected under the null model), while those below the line exhibit phylogenetic clumping (i.e., communities composed of oligotypes that are more related than expected under the null model)

likely to co-occur than expected by chance. This is a pattern that has been observed at broad scales across diverse communities from plants [71] to freshwater bacteria [72] and has frequently been interpreted as evidence for environmental filtering [52] but might also reflect local evolutionary radiations, in this case bacterial radiation within host guts [72]. The phylogenetic overdispersion we observed in the chimpanzee microbiomes-with individuals and groups sampling from overdispersed bacterial lineages -was thus unusual. Phylogenetic overdispersion has been suggested to be indicative of competitive displacement of closely related species and might indicate stronger interspecific competition between bacterial oligotypes in chimpanzees than monkeys. However, we found that patterns of overdispersion were strongest deeper in the bacterial phylogeny, and weaker towards the tips, where we might otherwise predict competition would be strongest. Further, for chimpanzees we did not see strong evidence for nesting and increasing the source pool did not expand the phylogenetic breadth of the gut bacterial community. The broad taxonomic dispersion of chimpanzee gut microbiomes is consistent with high exposure to diverse bacterial clades.

There are a number of aspects of chimpanzee ecology, life history, and physiology that might explain the unique microbiome community structure found in chimpanzees. For example, chimpanzees have the largest home ranges of any of the NHP included in the current study, potentially exposing them to a greater diversity of bacteria. Chimpanzees also diversify their diet to include more leaves during periods of fruit scarcity [73], potentially creating a niche for the same bacteria found in a folivore's microbiome. Mangabeys also include leaves from a number of plant species in their diet, so a similar shift in the microbiome might have been expected in this species as well [74]. Hunting of other NHP by chimpanzees could also be a 

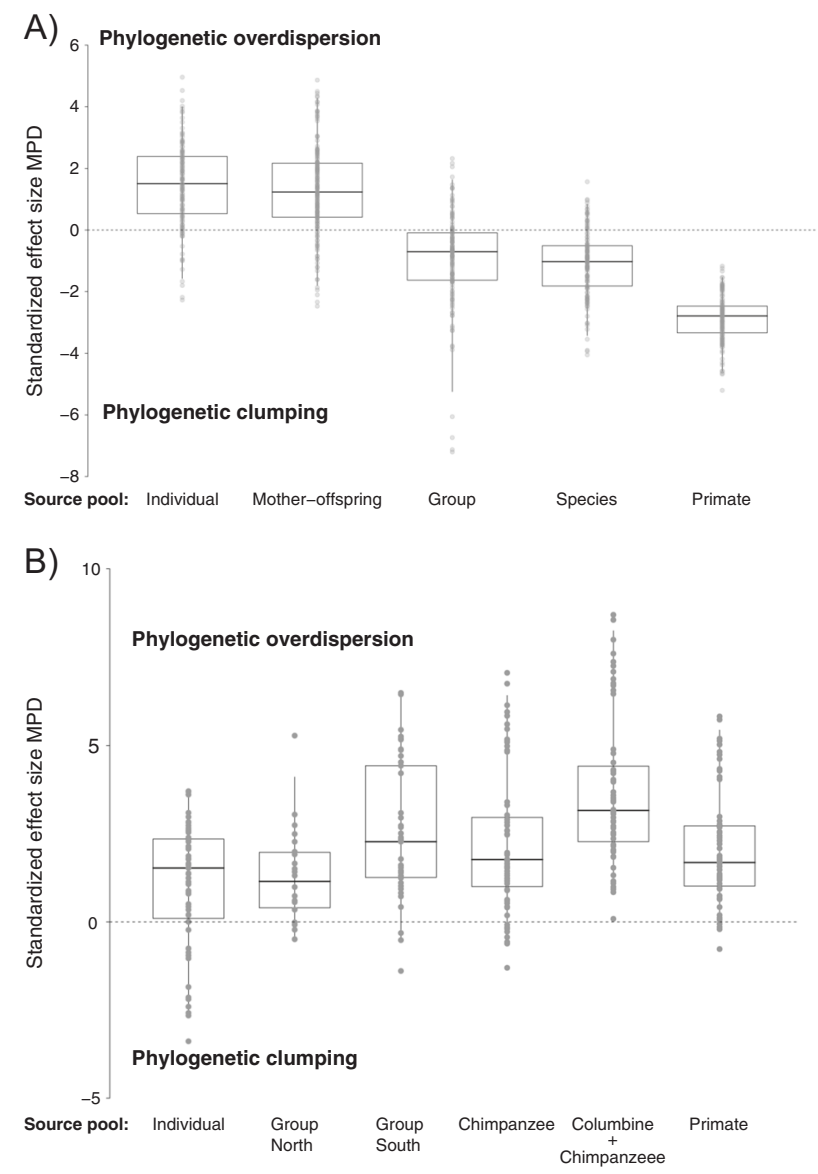

Fig. 5 Standardized effect size of mean phylogenetic distance based on null model simulations of the bacterial community in each fecal sample using different bacterial source pools. a For sooty mangabeys for each individual that was included in a mother-offspring pair, we used a source pool including only the bacteria found in any sample of a repeatedly sampled individual. We also considered a mother-offspring pool, using a source pool of only the bacteria found in any sample of the respective mother-offspring pair. For the group pool we used a source pool of only the bacteria found in the social group, for the host species, using a source pool of only the bacteria found in any sooty mangabey sample, and for the primate pool a source pool of all bacteria found in this study. b For chimpanzees we conducted a similar analysis; we did not include mother-offspring pairs and rather conducted the group analysis for each of the two groups for which we had repeated sampling of individuals. In addition, we included a source pool level that consisted of bacteria found in any colobine or chimpanzee sample. The solid middle horizontal line of the rectangles represents the median, the rectangle shows the quartiles, and the vertical line represents the 2.5 th and 97.5 th percentiles, while the values for each sample are indicated by overlapping gray circles. Values above the dashed line are those exhibiting phylogenetic overdispersion while those below the line exhibit phylogenetic clumping

route by which chimpanzees have been exposed to high bacterial diversity.

While the observed incongruence between microbial communities and NHP evolutionary relationships supports the hunting hypothesis, it warrants additional study as longbranch attraction presents difficulties for comparing such distinct bacterial communities [75]. The inclusion of an outgroup that is also present in this ecosystem (e.g., a galago) in future analyses might help to better resolve these relationships, as would a more in-depth sampling of colobines in this ecosystem. The overdispersion of bacterial lineages within chimpanzee individuals and social groups does not seem to be driven by recent consumption of colobine prey, as those samples containing colobine DNA were not unique in being overdispersed nor in their similarity to colobine samples. Stomach acidity has been hypothesized to serve as a barrier to bacterial colonization of the gut, particularly the high acidity found in the stomachs of carnivores and scavengers [76]. This might make bacterial establishment of monkey prey bacteria in an individual and subsequently into its social group an extremely rare event, mirroring the usually low rate of crossspecies viral transmission in this ecosystem [59, 77-79]. Many aspects of chimpanzee ecology, life history, and physiology likely act in concert to influence aspects of their unique microbiome community structure and future studies are needed to disentangle the multitude of factors influencing microbiome assembly. Fahy et al. suggested that individual chimpanzees differ in their consumption of different types of foods, including colobines [80], and future work including detailed dietary information from individual chimpanzees will enable an examination of the role of dietary variation in shaping their gut microbiomes.

\section{Conclusion}

We present a comprehensive analysis of contemporaneous microbiomes of sympatric NHP. Despite sharing an environment and exposure to a similar bacterial source pool, we found that host species maintained distinct microbiomes. Our findings support the idea that beyond the importance of genetic factors, combined with anatomy, physiology, and life history, differential exposure from conspecifics is important in influencing the gut microbiome of NHP. We found that individuals had persistent microbiomes influenced by social group and transiently by birth, but surprisingly not by grooming and proximity. Contrary to phylogenetic expectations, gut microbiomes of chimpanzees clustered with their colobine prey, an even more remarkable finding given the specialized diet of the latter, which has been accompanied by physiological and microbial adaptations enabling foregut fermentation. In contrast to signals suggesting phylogenetic clustering in the microbiomes of monkeys, chimpanzee microbiomes also exhibited phylogenetic overdispersion, suggesting a unique ecological process impacts their community assembly. While we detected differences in the microbiome between individuals, groups, and species, an exciting avenue of 
research is to determine the functional implications of these differences. Metatranscriptomics and metaproteomics provide exciting approaches for assessing functional diversity in the microbiome of different individuals and species [81]. Further studies are required to understand the aspects of chimpanzee ecology, life history, and physiology that explain their uniquely overdispersed microbiome community structure.

Acknowledgements This article represents a chapter in the doctoral dissertation of J.F.G. and benefited greatly from the input of his supervision committee, David Marcogliese, Charles Nunn, and Louis Lefebvre. We are grateful to the Ivorian Ministry of Research, the Ivorian Ministry of Environment and Forests, as well as the Office Ivoirien des Parcs et Réserves for giving permission to conduct this study. We thank the directorship of the Taï National Park, the Centre Suisse de Recherche Scientifique, the Taï Chimpanzee Project and their teams of field assistants for their support. J.F.G. was supported by an NSF Graduate Research Fellowship (DGE-1142336), the Canadian Institutes of Health Research's Strategic Training Initiative in Health Research's Systems Biology Training Program, an NSERC Vanier Canada Graduate Scholarship (CGS), and a long-term Research Grant from the German Academic Exchange Service (DAAD-9152583757048249). Core-funding for the Taï Chimpanzee Project is provided by the Max Planck Society. This research also benefited from discussions within the Deutsche Forschungsgemeinschaft (DFG) research group "Sociality and Health in Primates" (FOR2136). For assistance in the field we thank Ariane Düx, Vera Leinert, Bekah Myers, Jonathan Müller-Tiburtius, Sylvain Lemoine, and the long-term mangabey field assistants Simon T. Kannieu, Daniel Bouin, and Gnimion Florent. For assistance and support with sequencing at the Microbial Analysis and Services Facility at the University of Connecticut we are grateful to Kendra Maas. For their support in the laboratory, J.F.G. thanks Ulla Thiesen, Kevin Merkel, Andreas Sachse, and the P3 group at the Robert Koch Institute. J.F.G. thanks James Herrera and Will Pearse for discussions and sharing $\mathrm{R}$ code for phylogenetic analyses.

\section{Compliance with ethical standards}

Conflict of interest The authors declare that they have no conflict of interest.

\section{References}

1. Tremaroli V, Bäckhed F. Functional interactions between the gut microbiota and host metabolism. Nature. 2012;489:242-9.

2. Collins SM, Surette M, Bercik P. The interplay between the intestinal microbiota and the brain. Nat Rev Microbiol. 2012;10:735-42.

3. Hooper LV, Littman DR, Macpherson AJ. Interactions between the microbiota and the immune system. Science. 2012;336:1268-73.

4. Ezenwa VO, Williams AE. Microbes and animal olfactory communication: where do we go from here? Bioessays. 2014;36:847-54.

5. Bordenstein SR, Theis KR. Host biology in light of the microbiome: ten principles of holobionts and hologenomes. PLoS Biol. 2015;13:e1002226.

6. Kopac SM, Klassen JL. Can they make it on their own? Hosts, microbes, and the holobiont niche. Front Microbiol. 2016;7:1647.

7. Zilber-Rosenberg I, Rosenberg E. Role of microorganisms in the evolution of animals and plants: the hologenome theory of evolution. FEMS Microbiol Rev. 2008;32:723-35.
8. Gogarten JP, Doolittle WF, Lawrence JG. Prokaryotic evolution in light of gene transfer. Mol Biol Evol. 2002;19:2226-38.

9. Theis KR, Dheilly NM, Klassen JL, Brucker RM, Baines JF, Bosch TCG et al. Getting the hologenome concept right: an ecoevolutionary framework for hosts and their microbiomes. mSystems. 2016;1:e00028-16.

10. Ochman H, Worobey M, Kuo C-H, Ndjango J-BN, Peeters M, Hahn $\mathrm{BH}$, et al. Evolutionary relationships of wild hominids recapitulated by gut microbial communities. PLoS Biol. 2010;8: e1000546.

11. Moeller AH, Caro-Quintero A, Mjungu D, Georgiev AV, Lonsdorf EV, Muller MN, et al. Cospeciation of gut microbiota with hominids. Science. 2016;353:380-2.

12. Groussin M, Mazel F, Sanders JG, Smillie CS, Lavergne S, Thuiller W, et al. Unraveling the processes shaping mammalian gut microbiomes over evolutionary time. Nat Commun. 2017;8:14319.

13. Ventura M, Turroni F, Motherway MOC, MacSharry J, van Sinderen D. Host-microbe interactions that facilitate gut colonization by commensal bifidobacteria. Trends Microbiol. 2012;20:467-76.

14. Stevens CE, Hume ID. Contributions of microbes in vertebrate gastrointestinal tract to production and conservation of nutrients. Physiol Rev. 1998;78:393-427.

15. Gritz EC, Bhandari V. The human neonatal gut microbiome: a brief review. Front Pediatr. 2015;3:17.

16. Dominguez-Bello MG, Costello EK, Contreras M, Magris M, Hidalgo G, Fierer N, et al. Delivery mode shapes the acquisition and structure of the initial microbiota across multiple body habitats in newborns. Proc Natl Acad Sci. 2010;107:11971-5.

17. Tung J, Barreiro LB, Burns MB, Grenier J-C, Lynch J, Grieneisen LE, et al. Social networks predict gut microbiome composition in wild baboons. eLife. 2015;4:e05224.

18. Gogarten JF, Akoua-Koffi C, Calvignac-Spencer S, Leendertz SAJ, Weiss S, Couacy-Hymann E, et al. The ecology of primate retroviruses-an assessment of 12 years of retroviral studies in the Taï national park area, Côte d'Ivoire. Virology. 2014;460: $147-53$.

19. Goldberg TL, Gillespie TR, Rwego IB, Wheeler E, Estoff EL, Chapman CA. Patterns of gastrointestinal bacterial exchange between chimpanzees and humans involved in research and tourism in western Uganda. Biol Conserv. 2007;135:511-7.

20. Moeller AH, Peeters M, Ndjango J-B, Li Y, Hahn BH, Ochman H. Sympatric chimpanzees and gorillas harbor convergent gut microbial communities. Genome Res. 2013;23:1715-20.

21. Chivers DJ, Hladik CM. Morphology of the gastrointestinal tract in primates: comparisons with other mammals in relation to diet. $\mathrm{J}$ Morphol. 1980;166:337-86.

22. Boesch C. Chimpanzees-red colobus monkeys: a predator-prey system. Anim Behav. 1994;47:1135-48.

23. Boesch C, Achermann HB. The chimpanzees of the Taï forest: behavioural ecology and evolution. Oxford University Press: Oxford and New York; 2000.

24. Range F, Noë R. Familiarity and dominance relations among female sooty mangabeys in the Taï National Park. Am J Primatol. 2002;56:137-53.

25. Wittig RM, Boesch C. Food competition and linear dominance hierarchy among female chimpanzees of the Tai National Park. Int J Primatol. 2003;24:847-67.

26. De Nys HM, Madinda NF, Merkel K, Robbins M, Boesch C, Leendertz $\mathrm{FH}$, et al. A cautionary note on fecal sampling and molecular epidemiology in predatory wild great apes. Am J Primatol. 2015;77:833-40.

27. Minhós T, Nixon E, Sousa C, Vicente LM, da Silva MF, Sá R, et al. Genetic evidence for spatio-temporal changes in the dispersal patterns of two sympatric African colobine monkeys. Am J Phys Anthropol. 2013;150:464-74. 
28. Schubert G, Stockhausen M, Hoffmann C, Merkel K, Vigilant L, Leendertz FH, et al. Targeted detection of mammalian species using carrion fly-derived DNA. Mol Ecol Resour. 2015;15: 285-94.

29. Calvignac-Spencer S, Merkel K, Kutzner N, Kühl H, Boesch C, Kappeler PM, et al. Carrion fly-derived DNA as a tool for comprehensive and cost-effective assessment of mammalian biodiversity. Mol Ecol. 2013;22:915-24.

30. Taylor PG. Reproducibility of ancient DNA sequences from extinct Pleistocene fauna. Mol Biol Evol. 1996;13:283-5.

31. Altschul SF, Gish W, Miller W, Myers EW, Lipman DJ. Basic local alignment search tool. J Mol Biol. 1990;215:403-10.

32. Nelson MC, Morrison HG, Benjamino J, Grim SL, Graf J. Analysis, optimization and verification of Illumina-generated $16 \mathrm{~S}$ rRNA gene amplicon surveys. PLoS ONE. 2014;9:e94249.

33. Caporaso JG, Lauber CL, Walters WA, Berg-Lyons D, Huntley J, Fierer N, et al. Ultra-high-throughput microbial community analysis on the Illumina HiSeq and MiSeq platforms. ISME J. 2012. https://doi.org/10.1038/ismej.2012.1038

34. Callahan BJ, McMurdie PJ, Rosen MJ, Han AW, Johnson AJA, Holmes SP. DADA2: high-resolution sample inference from Illumina amplicon data. Nat Methods. 2016;13:581-3.

35. Callahan BJ, Sankaran K, Fukuyama JA, McMurdie PJ, Holmes SP. Bioconductor workflow for microbiome data analysis: from raw reads to community analyses. F1000Res. 2016;5:1492.

36. DeSantis TZ, Hugenholtz P, Larsen N, Rojas M, Brodie EL, Keller K, et al. Greengenes, a chimera-checked 16S rRNA gene database and workbench compatible with ARB. Appl Environ Microbiol. 2006;72:5069-72.

37. Schliep KP. phangorn: phylogenetic analysis in R. Bioinformatics. 2010;btq706.

38. $\mathrm{R}$ Core Team. R: A language and environment for statistical computing. Vienna, Austria: R Foundation for Statistical Computing; 2015. https://www.R-project.org/

39. Lozupone C, Lladser ME, Knights D, Stombaugh J, Knight R. UniFrac: an effective distance metric for microbial community comparison. ISME J. 2011;5:169.

40. Bray JR, Curtis JT. An ordination of the upland forest communities of southern Wisconsin. Ecol Monogr. 1957;27:325-49.

41. McMurdie PJ, Holmes S. phyloseq: an R package for reproducible interactive analysis and graphics of microbiome census data. PLoS ONE. 2013;8:e61217.

42. Sokal RR, Rohlf FJ. Biometry: the principles and practice of statistics in biological research. 3rd ed. New York: W. H. Freeman and Company; 1995.

43. Siegel S, Castellan NJ. Nonparametric statistics for the behavioral sciences. New York: McGraw-Hill; 1988.

44. McCullagh P, Nelder JA. Generalized linear models. London: Chapman and Hall; 1996.

45. Baayen RH. Analyzing linguistic data: a practical introduction to statistics using R. Cambridge University Press: Cambridge; 2008.

46. Bates D, Mächler M, Bolker B, Walker S. Fitting linear mixedeffects models using lme4. J Stat Softw. 2015;67:1-48.

47. Forstmeier W, Schielzeth H. Cryptic multiple hypotheses testing in linear models: overestimated effect sizes and the winner's curse. Behav Ecol Sociobiol. 2011;65:47-55.

48. Dobson AJ, Barnett A. An introduction to generalized linear models. CRC Press: Boca Raton, FL; 2008.

49. Swofford DL. PAUP*. Phylogenetic analysis using Parsimony (*and other methods). Sunderland, Massachusetts: Sinauer Associates; 2002.

50. Rambaut A, Lam TT, Max Carvalho L, Pybus OG. Exploring the temporal structure of heterochronous sequences using TempEst (formerly Path-O-Gen). Virus Evol. 2016;2:vew007.

51. Huson DH, Bryant D. Application of phylogenetic networks in evolutionary studies. Mol Biol Evol. 2006;23:254-67.
52. Webb CO, Ackerly DD, McPeek MA, Donoghue MJ. Phylogenies and community ecology. Annu Rev Ecol Syst. 2002;33: 475-505.

53. Erickson DL, Jones FA, Swenson NG, Pei N, Bourg NA, Chen $\mathrm{W}$, et al. Comparative evolutionary diversity and phylogenetic structure across multiple forest dynamics plots: a mega-phylogeny approach. Front Genet. 2014;5:358.

54. Pearse WD, Cadotte MW, Cavender-Bares J, Ives AR, Tucker CM, Walker SC, et al. pez: phylogenetics for the environmental sciences. Bioinformatics. 2015;31:2888-90.

55. Kembel SW, Cowan PD, Helmus MR, Cornwell WK, Morlon H, Ackerly DD, et al. Picante: R tools for integrating phylogenies and ecology. Bioinformatics. 2010;26:1463-4.

56. Fogel AT. The gut microbiome of wild lemurs: a comparison of sympatric Lemur catta and Propithecus verreauxi. Folia Primatol. 2015;86:85-95.

57. McCord AI, Chapman CA, Weny G, Tumukunde A, Hyeroba D, Klotz K, et al. Fecal microbiomes of non-human primates in western Uganda reveal species-specific communities largely resistant to habitat perturbation. Am J Primatol. 2014;76:347-54.

58. Boesch C, Boesch H. Hunting behavior of wild chimpanzees in the Taï National Park. Am J Phys Anthropol. 1989;78:547-73.

59. Leendertz SAJ, Locatelli S, Boesch C, Kücherer C, Formenty P, Liegeois $\mathrm{F}$, et al. No evidence for transmission of SIVwrc from western red colobus monkeys (piliocolobus badius badius) to wild west african chimpanzees (pan troglodytes verus) despite high exposure through hunting. BMC Microbiol. 2011;11:24.

60. Eppinger M, Baar C, Linz B, Raddatz G, Lanz C, Keller H, et al. Who ate whom? adaptive Helicobacter genomic changes that accompanied a host jump from early humans to large felines. PLoS Genet. 2006;2:e120.

61. Faith JJ, Guruge JL, Charbonneau M, Subramanian S, Seedorf H, Goodman AL, et al. The long-term stability of the human gut microbiota. Science. 2013;341:1237439.

62. Ren T, Grieneisen LE, Alberts SC, Archie EA, Wu M. Development, diet and dynamism: longitudinal and cross-sectional predictors of gut microbial communities in wild baboons. Environ Microbiol. 2015;18:1312-25.

63. Aivelo T, Laakkonen J, Jernvall J. Population-and individual-level dynamics of the intestinal microbiota of a small primate. Appl Environ Microbiol. 2016;82:3537-45.

64. Moeller AH, Peeters M, Ayouba A, Ngole EM, Esteban A, Hahn $\mathrm{BH}$, et al. Stability of the gorilla microbiome despite simian immunodeficiency virus infection. Mol Ecol. 2015;24:690-7.

65. Degnan PH, Pusey AE, Lonsdorf EV, Goodall J, Wroblewski EE, Wilson ML, et al. Factors associated with the diversification of the gut microbial communities within chimpanzees from Gombe National Park. Proc Natl Acad Sci USA. 2012;109:13034-9.

66. Amato KR, Yeoman CJ, Kent A, Righini N, Carbonero F, Estrada A, et al. Habitat degradation impacts black howler monkey (Alouatta pigra) gastrointestinal microbiomes. ISME J. 2013;7: 1344-53.

67. Leibold MA, Holyoak M, Mouquet N, Amarasekare P, Chase JM, Hoopes MF, et al. The metacommunity concept: a framework for multi-scale community ecology. Ecol Lett. 2004;7:601-13.

68. Funkhouser LJ, Bordenstein SR. Mom knows best: the universality of maternal microbial transmission. PLoS Biol. 2013;11: e1001631.

69. Yatsunenko T, Rey FE, Manary MJ, Trehan I, Dominguez-Bello MG, Contreras $\mathrm{M}$, et al. Human gut microbiome viewed across age and geography. Nature. 2012;486:222.

70. Moeller AH, Foerster S, Wilson ML, Pusey AE, Hahn BH, Ochman H. Social behavior shapes the chimpanzee panmicrobiome. Sci Adv. 2016b;2:e1500997.

71. Kembel SW, Hubbell SP. The phylogenetic structure of a neotropical forest tree community. Ecology. 2006;87:S86-99. 
72. Horner-Devine MC, Bohannan BJM. Phylogenetic clustering and overdispersion in bacterial communities. Ecology. 2006;87:S100-8.

73. Doran D. Influence of seasonality on activity patterns, feeding behavior, ranging, and grouping patterns in Tai chimpanzees. Int $\mathbf{J}$ Primatol. 1997;18:183-206.

74. McGraw WS, Vick AE, Daegling DJ. Sex and age differences in the diet and ingestive behaviors of sooty mangabeys (Cercocebus atys) in the Tai forest, Ivory coast. Am J Phys Anthropol. 2011; 144:140-53.

75. Bergsten J. A review of long-branch attraction. Cladistics. 2005;21:163-93.

76. Beasley DE, Koltz AM, Lambert JE, Fierer N, Dunn RR. The evolution of stomach acidity and its relevance to the human microbiome. PLoS ONE. 2015;10:e0134116.

77. Anoh AE, Murthy S, Akoua-Koffi C, Couacy-Hymann E, Leendertz FH, Calvignac-Spencer S, et al. Cytomegaloviruses in a community of wild nonhuman primates in Taï National Park, Côte D'Ivoire. Viruses. 2017;10:11.
78. Leendertz FH, Zirkel F, Couacy-Hymann E, Ellerbrok H, Morozov VA, Pauli G, et al. Interspecies transmission of simian foamy virus in a natural predator-prey system. J Virol. 2008; 82:7741-4.

79. Murthy S, Couacy-Hymann E, Metzger S, Nowak K, De Nys H, Boesch $\mathrm{C}$, et al. Absence of frequent herpesvirus transmission in a nonhuman primate predator-prey system in the wild. J Virol. 2013;87:10651-9.

80. Fahy GE, Richards M, Riedel J, Hublin J-J, Boesch C. Stable isotope evidence of meat eating and hunting specialization in adult male chimpanzees. Proc Natl Acad Sci USA. 2013;110: 5829-33.

81. Bonin A, Taberlet P, Zinger L, Coissac E. Environmental DNA: for biodiversity research and monitoring. Oxford University Press: Oxford and New York; 2018.

82. Arnold C, Matthews LJ, Nunn CL. The 10kTrees website: a new online resource for primate phylogeny. Evol Anthropol. 2010;19: $114-8$. 